\title{
Formation of Lipid Peroxide in Doxapram-treated Mice
}

\author{
Ken-ichi Sasaki,* Shinobu Furusawa, and Gilchi Takayanagi \\ Cancer Research Institute, Tohoku College of Pharmaceutical \\ Sciences, 4-4-1 Komatsushima, Sendai, 983, Japan
}

(Received April 23, 1982)

\begin{abstract}
The effect of doxapram, a respiratory stimulant, on the formation of lipid peroxide was investigated in mice. A significant increase of lipid peroxide was observed in the liver after injection of doxapram $(100 \mathrm{mg} / \mathrm{kg}, i . p$.$) and the level of lipid peroxide was about$ 5 -fold greater than that of control mice at $4 \mathrm{~h}$ after injection. However, no increase of lipid peroxide was observed in the brain, heart, lung, spleen or kidney of mice treated with doxapram. On the other hand, doxapram initially increased the activity of xanthine oxidase but significantly reduced it from about $4 \mathrm{~h}$ after injection, and also reduced the activities of superoxide dismutase and glutathione peroxidase in the liver. The level of $\alpha$-tocopherol decreased slightly in the liver after administration of doxapram. This enhancement of lipid peroxidation in the liver in doxapram-treated mice suggests that an increase of superoxide anion and an inhibition of free radical scavenging reactions in vivo may be produced by the drug.
\end{abstract}

Keywords-doxapram; lipid peroxidation; mice; xanthine oxidase; superoxide dismutase; glutathione peroxidase; $\alpha$-tocopherol

The formation of lipid peroxides in vivo has aroused great interest in connection with aging phenomena and various diseases. Lipid peroxidation is regarded as a fundamental process in the development of some forms of tissue damage. Further degradation of membrane lipids and structural damage of membrane-bound enzymes may also result from this process. ${ }^{1)}$

Increases in lipid peroxidation usually cause a decrease in the ability to metabolize drugs, at least in part by promoting the degradation of cytochrome P-450 which is required for metabolism. ${ }^{2,3)}$ In the previous work, ${ }^{4,5)}$ we demonstrated that doxapram, a respiratory stimulant, suppressed the hepatic cytochrome P-450-dependent enzyme activities, and changed the action of coadministered drugs in mice.

The present investigation was initiated to clarify the mechanism of lipid peroxidation induced by doxapram in mouse liver.

\section{Materials and Methods}

Animals _- Male mice of ddY strain weighing 20-22 $\mathrm{g}$ were employed in all experiments. Food and water were given freely.

Drugs-Doxapram was purchased from Kissei Yakuhin Co. $\alpha$-Tocopherol was from Wako Junyaku Kogyo Co. Sodium pentobarbital was from Tokyo Kasei Co. Doxapram and sodium pentobarbital were each dissolved in $0.9 \%$ saline solution, while $\alpha$-tocopherol was suspended in $0.5 \%$ Tween 80 solution. Drugs were injected intraperitoneally.

Measurement of Lipid Peroxides_-Mice were sacrificed by decapitation and the tissues were excised. Ten percent tissue homogenates were prepared in $0.15 \mathrm{M} \mathrm{KCl}$, and used for assay. The lipid peroxide was estimated by measuring malonaldehyde production using a thiobarbituric acid method. ${ }^{6}$

Measurement of Enzyme Activity in Liver of Mice-The activity of superoxide dismutase was measured by the method of Beauchamp and Fridovich. ${ }^{\text {" }}$ The activity of glutathione peroxidase was measured according to the method of Stults et al. ${ }^{8)}$ The activity of xanthine oxidase was assayed by the method described previously. ${ }^{91}$ The activities of aminopyrine $N$-demethylase and aniline hydroxylase were measured according to the method of Kitagawa et al. ${ }^{10)}$ Protein concentration was determined by the method of Lowry et al. ${ }^{111}$ using crystalline bovine serum albumin as a standard.

Measurement of $\alpha$-Tocopherol-The level of $\alpha$-tocopherol in liver and plasma was determined by the method of Katsui. ${ }^{12)}$ 
Measurement of Duration of Sleep_-After intraperitoneal administration of $50 \mathrm{mg} / \mathrm{kg}$ of pentobarbital, the period of disappearance of the righting reflex in mice was measured to estimate the duration of sleep. Doxapram was administered intraperitoneally $60 \mathrm{~min}$ prior to the injection of pentobarbital.

Statistical Evaluation-_- - Statistical significance was calculated by using Student's $t$-test.

\section{Results}

\section{Effect of Doxapram on Lipid Peroxide Formation}

As shown in Fig. 1, an enhancement of lipid peroxidation in mouse liver was observed after intraperitoneal administration of doxapram. The formation of lipid peroxide after 50 or $100 \mathrm{mg} / \mathrm{kg}(i . p$.$) of doxapram reached a maximum of 375.0 \%$ or $538.7 \% 2$ or $4 \mathrm{~h}$ later, respectively, as compared with that of control mice, and then returned to the normal range 4 or $8 \mathrm{~h}$ later.

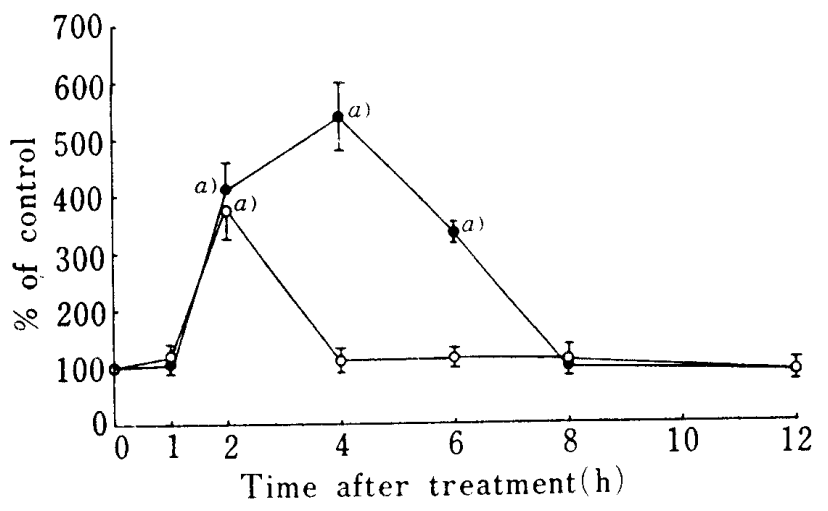

Fig. 1. Time Course of Lipid Peroxide Level in Mouse Liver after Doxapram Injection

Mice were injected with doxapram (50 or $100 \mathrm{mg} / \mathrm{kg}, i . p$. ) and lipid peroxides of the liver were measured at various times after treatment. Each group comprised seven mice. Data are expressed as per cent of the control. The control value (mean $t S$. E.) was $160.1 \pm 5.3 \mathrm{nmol}$ malonaldehyde/g of liver. - - : doxapram 50 $\mathrm{mg} / \mathrm{kg}$. - - : doxapram $100 \mathrm{mg} / \mathrm{kg}$. Significant differences from the control value are indicated by $a)(p<0.01)$.

On the other hand, pretreatment with the antioxidant $\alpha$-tocopherol $(50 \mathrm{mg} / \mathrm{kg}, i . p$.) $30 \mathrm{~min}$ prior to doxapram completely suppressed the formation of lipid peroxide by doxapram (100 $\mathrm{mg} / \mathrm{kg}, i . p$.$) . The measurement was usually carried out 4 \mathrm{~h}$ after doxapram injection. That is to say, the mean values $\pm \mathrm{S}$.E. of untreated mice, doxapram-treated mice and $\alpha$-tocopherol plues doxapram-treated mice were $160.1 \pm 15.3,862.5 \pm 89.5$ and $146.7 \pm 9.5 \mathrm{nmol}$ of malonaldehyde/g of liver, respectively. Nevertheless, the decrease of the activities of aminopyrine $\mathrm{N}$ demethylase and aniline hydroxylase in the liver, and the prolongation of pentobarbital sleeping time after treatment with doxapram $(25$ or $100 \mathrm{mg} / \mathrm{kg}, i . p$.) were not inhibited by pretreatment with $\alpha$-tocopherol $(50 \mathrm{mg} / \mathrm{kg}$, i.p.) $30 \mathrm{~min}$ prior to doxapram. The mean activities $\pm \mathrm{S}$.E. of aminopyrine $N$-demethylase and aniline hydroxylase were $2.12 \pm 0.10$ and $2.17 \pm 0.11 \mathrm{nmol} / 30$ $\mathrm{min} / \mathrm{mg}$ of protein in untreated mice, $1.35 \pm 0.09$ and $1.66 \pm 0.07 \mathrm{nmol} / 30 \mathrm{~min} / \mathrm{mg}$ protein in doxapram-treated mice and $1.45 \pm 0.08$ and $1.45 \pm 0.09 \mathrm{nmol} / 30 \mathrm{~min} / \mathrm{mg}$ of protein in $\alpha$-tocopherol plus doxapram-treated mice, respectively. The mean sleeping time \pm S.E. induced by pentobarbital was $52.2 \pm 4.3 \mathrm{~min}$ in untreated mice, $180.1 \pm 20.7 \mathrm{~min}$ in doxapram-treated mice, and $207.0 \pm 17.8 \mathrm{~min}$ in $\alpha$-tocopherol plus doxapram-treated mice, respectively.

The effect of doxapram on the formation of lipid peroxide in various other tissues was examined and the results are shown in Table I. There was no significant effect of doxapram $(100 \mathrm{mg} / \mathrm{kg}, i . p$.$) on the formation of lipid peroxide in the brain, heart, lung, spleen or kidney$ 
TABle I. Effect of Doxapram on Lipid Peroxide in Various Tissues (nmol Malonaldehyde/g of Tissue)

\begin{tabular}{lcc}
\hline Tissue & Control & Doxapram \\
\hline Brain & $209.3 \pm 16.6$ & $232.9 \pm 19.0$ \\
Heart & $218.7 \pm 15.1$ & $227.8 \pm 15.3$ \\
Lung & $199.2 \pm 16.3$ & $224.6 \pm 18.1$ \\
Spleen & $187.7 \pm 12.0$ & $206.4 \pm 19.3$ \\
Kidney & $217.5 \pm 18.4$ & $240.4 \pm 18.5$ \\
Liver & $160.1 \pm 15.3$ & $862.5 \pm 89.5^{a)}$ \\
\hline
\end{tabular}

Mice were injected with doxapram $(100 \mathrm{mg} / \mathrm{kg}, i . p$.$) and lipid peroxides in various tissues were$ measured $4 \mathrm{~h}$ later. Each value is the mean of six to seven experiments and data are expressed as mean $t$ S.E. Signiticant difference from the control value is indicated by a) $(p<1,01)$.

th after treatment. Thus, doxapram produced a significant increment in lipid peroxide only in the liver.

\section{Effect of Doxapram on the Activities of Superoxide Dismutase, Glutathione Peroxidase and Xanthine Oxidase}

Figure 2 shows the time course of the three enzyme activities after the doxapram treatment $(100 \mathrm{mg} / \mathrm{kg}, i . p$.$) . The activity of superoxide dismutase in the liver decreased from 1$ to $8 \mathrm{~h}$ after doxapram and the activity of glutathione peroxidase in the liver decreased from 1 to $4 \mathrm{~h}$ after doxapram. In contrast, the activity of xanthine oxidase in the liver increased significantly from 1 to $2 \mathrm{~h}$ after doxapram and returned to the normal level $+\mathrm{h}$ after, then decreased from 6 to $12 \mathrm{~h}$ after doxapram.

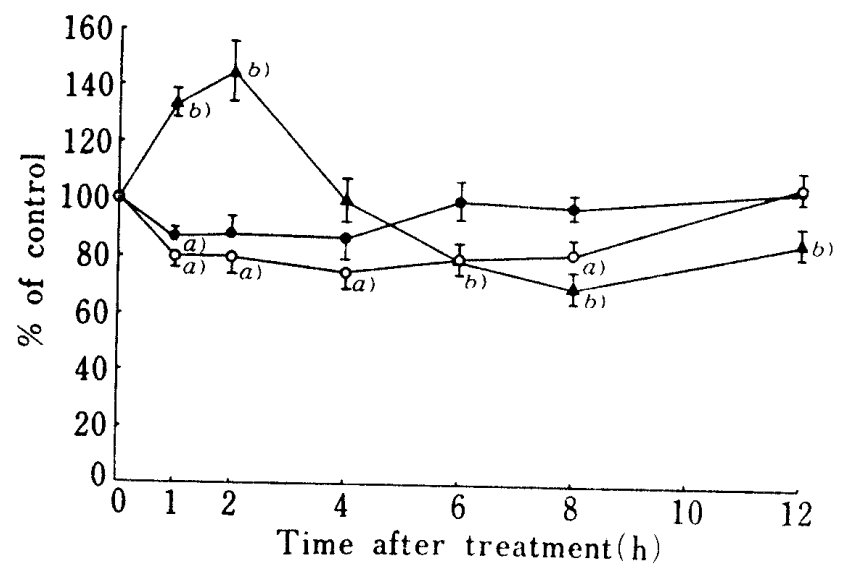

Fig. 2. Time Course of the Activities of Superoxide Dismutase, Glutathione Peroxidase and Xanthine Oxidase in Mouse Liver after Doxapram Injection

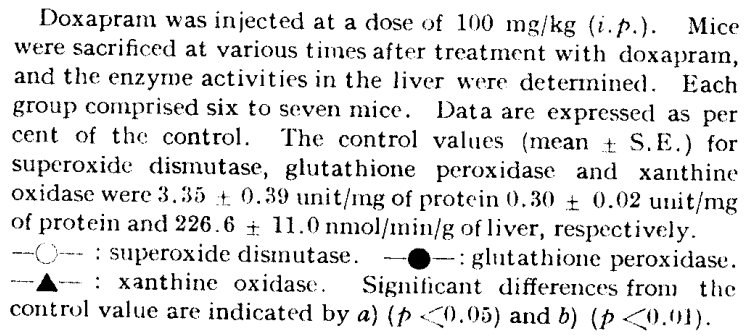
were sacrificed at various times after treatment with doxayram, and the enzyme activities in the liver were determined. Each group comprised six to seven mice. Dat a are expressed as per cent of the control. The control values (mean $t$ S.E.) for superoxide dismutase, glutathione peroxidase and xanthine oxidase were $3.35 \pm 0.39 \mathrm{unit} / \mathrm{mg}$ of protein $0.30 \pm 0.02 \mathrm{unit} / \mathrm{mg}$ of protein and $226.6+11.0 \mathrm{nmol} / \mathrm{min} / \mathrm{g}$ of liver, respectively - C- : superoxide dismutase. - - : glutathione peroxidase. A- : xanthine oxidase. Significant differences from the control value are indicated by $a)(p<0.05)$ and $b)(p<0.01)$.

\section{Effect of Doxapram on the Level of $\alpha$-Tocopherol in the Liver and Plasma}

The levels of $\alpha$-tocopherol in the liver and plasma were determined in untreated mice and doxapram-treated mice and the results are shown in Fig. 3. No great change was observed in 
doxapram-treated mice, though there was a slight decrease in $\alpha$-tocopherol in the liver $1 \mathrm{~h}$ after the injection of doxapram $(100 \mathrm{mg} / \mathrm{kg}, i . p$.$) .$

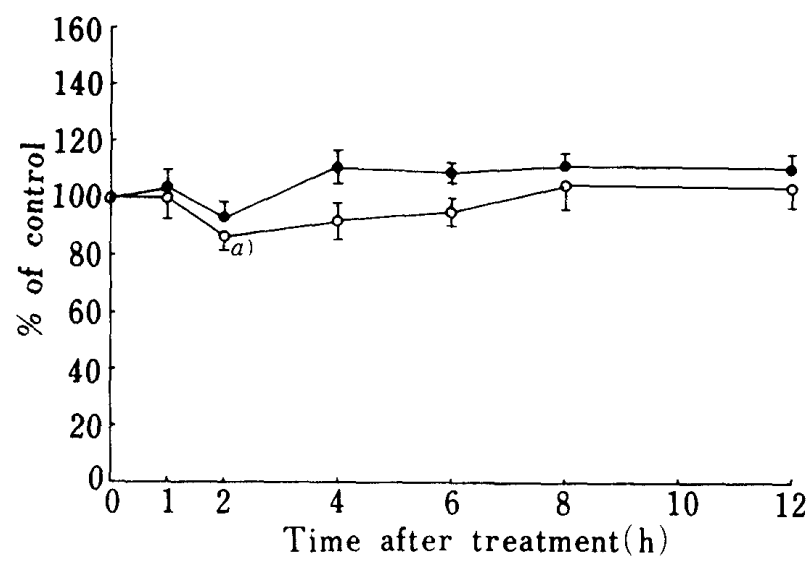

Fig. 3. Time Course of $\alpha$-Tocopherol Level in Mouse Liver and Plasma after Doxapram Injection

Doxapram was injected at a dose of $100 \mathrm{mg} / \mathrm{kg}(i . p$.$) . Each$ group comprised eight mice. Data are expressed as per cent of the control. The control values (mean \pm S.E.) for $\alpha$-tocopherol concentration in liver and plasma were $18.26 \pm 0.61 \mu \mathrm{g} / \mathrm{g}$ of liver and $4.25 \pm 0.13 \mu \mathrm{g} / \mathrm{ml}$ of plasma. - - : liver.

plasma. Significant difference from the control value is indicated by $a)(p<0.05)$.

\section{Discussion}

In recent years, dose-related effects of various drugs on lipid peroxidation, ${ }^{13,14}$ and an inverse relation between the formation of peroxide and the drug-metabolizing enzyme activity in the liver have been demonstrated by many investigators. ${ }^{15)}$ The decrease in drug-metabolizing activity due to cytochrome destruction as a result of lipid peroxidation has been well documented. ${ }^{16)}$

We previously reported on the mechanism of the interaction between doxapram and combined drugs, and showed that doxapram induced a depression of the hepatic microsomal drug-metabolizing system and consequently affected the drug level in the plasma. ${ }^{4,5}$ The objective of the present investigation was to study further the relation between lipid peroxidation and drug metabolism in mouse liver.

The formation of lipid peroxide in the liver was observed after the administration of doxapram to mice. The level of lipid peroxide after doxapram (50 or $100 \mathrm{mg} / \mathrm{kg}, i . p$.) reached a maximum 2 or $4 \mathrm{~h}$ later, respectively. No effect of doxapram on lipid peroxidation was observed in the brain, heart, lung, spleen or kidney, Thus, doxapram caused a significant increase of lipid peroxidation only in the liver. The results are in good accord with the depression of the hepatic microsomal drug-metabolizing system described previously. ${ }^{4,5}$ ) On the other hand, lipid peroxidation induced by doxapram was significantly inhibited by pretreatment with $\alpha$-tocopherol, which is an antioxidant and a free radical scavenger.

The superoxide anion is an important intermediate in the formation of singlet oxygen and hydroxyl radical, which are capable of initiating lipid peroxidation. It is well known that superoxide anion is generated by the xanthine-xanthine oxidase system ${ }^{17}$ and NADPHcytochrome $c$ reductase. ${ }^{18}$ In doxapram-treated mice, the activity of xanthine oxidase in the liver was markedly enhanced prior to the formation of lipid peroxide. It was reported in the previous paper ${ }^{5)}$ that the activity of NADPH-cytochrome c reductase was inhibited by the injection of doxapram. This finding suggests that NADPH-cytochrome c reductase is not involved in lipid peroxidation induced by doxapram. 
The role of superoxide dismutase and glutathione peroxidase in protection from lipid peroxidation in vivo is very important. Namely, the former scavenges superoxide anion in vivo, and the latter catalyzes the breakdown of hydrogen peroxide through the glutathione shuttle system and reduces lipid hydroperoxides. The activities of superoxide dismutase and glutathione peroxidase in the liver decreased after treatment with doxapram. These results therefore provide clear evidence for the mechanism of formation of lipid peroxide. The findings that in doxapram-treated mice the level of $\alpha$-tocopherol decreased slightly in the liver and the enhancement of peroxide was significantly inhibited by $\alpha$-tocopherol are also significant. In practice, however, pretreatment with $\alpha$-tocopherol did not inhibit the doxapram-induced suppression in the activities of aminopyrine $N$-demethylase and aniline hydroxylase or the doxapram-induced prolongation of pentobarbital sleeping time.

Therefore, it is considered that the formation of lipid peroxide after doxapram administration is probably due to the enhancement of superoxide anion formation and the inhibition of free radical scavenging systems in vivo.

\section{References and Notes}

1) W-M. Tom and M.R. Montgomery, Biochem. Pharmacol., 29, 3239 (1980).

2) E.D. Wills, Biochem. J., 123, 983 (1971).

3) B.A. Schacter, H.S. Marver, and V.A. Meyer, Biochim. Biophys. Acta, 279, 221 (1972).

4) K. Sasaki, S. Furusawa, and G. Takayanagi, J. Pharm Dyn., 4, 394 (1981).

5) K. Sasaki, S. Furusawa, and G. Takayanagi, Jpn. J. Pharmacol., 32, 699 (1982).

6) K. Yagi, Biochem. Med., 15, 212 (1976).

7) C. Baeuchamp and I. Fridovich, Anal. Biochem., 44, 276 (1971).

8) F.H. Stults, J.W. Forstrom, 1.'T.Y. Chiu, and A.L. Tappel, Arch. Biochem. Biophys., 183, 490 (1977).

9) K. Sasaki, S. Furusawa, and G. Takayanagi, Chem. Pharm. Bull., 29, 276 (1981).

10) H. Kitagawa, S. Yoshida, and T. Kamataki, Yakugaku Zasshi, 88, 954 (1968).

11) O.H. Lowry, N.J. Rosebrough, A.L. Farr, and R.J. Randall, J. Biol. Chem., 193, 265 (1951).

12) G. Katsui, Vitamins, 54, $361(1980)$.

13) K. Aikawa, T. Satoh, K. Kobayashi, and H. Kitagawa, J. Pharm. Dyn., 1, 22 (1978).

14) C.E. Myers, W.P. Mcciuire, R.H. Liss, I. Ifrim, K. Grotzinger, and R.C. Young, Scis).

15) T. Kamataki and H. Kitagawa, Biochem. Pharmacol. 22, 3199 (1973). 16) W. Levin, A.Y.H. Lu, M. Jacobson, R. Kuntzman, J.L. Poyer, and P.B. McCay, Arch. Biochem. Biophys.,
158, 842 (1973).

17) E.W. Kellogg and I. Fridovich, J. Biol. Chem., 250, 8812 (1975).

18) T.C. Pederson and S.D. Aust, Biochem. Biophys. Res. Commun., 48, 789 (1972). 